\title{
Effect of Exercise on NAFLD and Its Risk Factors: Comparison of Moderate versus Low Intensity Exercise
}

\author{
Preetam Nath ${ }^{1}$, Manas Kumar Panigrahi ${ }^{2}$, Manoj Kumar Sahu ${ }^{3}$, Jimmy Narayan ${ }^{3}$, \\ Ranjan Kumar Sahoo ${ }^{4}$, Ananya Apurba Patra ${ }^{4}$, Satyaswarup Jena ${ }^{5}$, Arun Kumar Patnaik ${ }^{6}$, \\ Anjan Jena ${ }^{6}$ and Shivaram Prasad Singh*7'
}

${ }^{1}$ Department of Gastroenterology \& Hepatology, Kalinga Institute of Medical Sciences, Bhubaneswar, Odisha, India; ${ }^{2}$ Department of Gastroenterology, All India Institute of Medical Sciences, Bhubaneswar, Odisha, India; ${ }^{3}$ Department of Gastroenterology, IMS and SUM Hospital, Bhubaneswar, Odisha, India; ${ }^{4}$ Department of Radiodiagnosis \& Imaging, Kalinga Institute of Medical Sciences, Bhubaneswar, Odisha, India; ${ }^{5}$ Department of Radiology, IMS and SUM Hospital, Bhubaneswar, Odisha, India; ${ }^{6}$ Dispensary, Biju Patnaik State Police Academy, Bhubaneswar, Odisha, India; ${ }^{7}$ Department of Gastroenterology, S.C.B. Medical College, 753007 Cuttack, Odisha, India

\begin{abstract}
Background and Aims: Lifestyle (exercise and dietary) modification is the mainstay of treatment for non-alcoholic fatty liver disease (NAFLD). However, there is paucity of data on effect of intensity of exercise in management of NAFLD, and we aimed to study the effect of variable intensities of exercise on NAFLD. Methods: The study was performed in the Department of Gastroenterology of the SCB Medical College, Cuttack and the Biju Patnaik State Police Academy, Bhubaneswar. The subjects were police trainees [18 in a moderate intensity exercise group (MIG) and 19 in a low intensity exercise group (LIG)] recruited for a 6 -month physical training course (261.8 Kcalorie, 3.6 metabolic equivalent in MIG and 153.6 Kcalorie, 2.1 metabolic equivalent in LIG). NAFLD was diagnosed by ultrasonography, with exclusion of all secondary causes of steatosis. All participants were evaluated by anthropometry (weight, height, body mass index (BMI), waist circumference), assessed for blood pressure and biochemical parameters (blood glucose, liver function test, lipid profile, serum insulin), and subjected to transabdominal ultrasonography before and after 6 months of physical training, and the results were compared. Results: Both the groups had similar BMI, fasting plasma glucose, AST, gammaglutamyl transpeptidase, insulin, and homeostatic model assessment-insulin resistance (known as HOMA-IR) $(p>0.05)$. However, subjects in the LIG were older and had lower alanine transaminase, higher triglycerides and lower high-density lipoproteins than MIG subjects. There was a significant reduction in BMI $(27.0 \pm 2.1$ to $26.8 \pm 2.0 ; p=0.001)$, fasting blood glucose $(106.7 \pm 21.6$ to $85.8 \pm 19.0 ; p<0.001)$, serum triglycerides $(167.5 \pm 56.7$ to $124.6 \pm 63.5 ; p=0.017)$, total choles-
\end{abstract}

Keywords: Exercise; NAFLD; Intensity; Physical activity.

Abbreviations: $1 \mathrm{H}-\mathrm{MRS}$, magnetic resonance spectroscopy; ALT, alanine transaminase; APRI, AST to platelet ratio; AST, aspartate transaminase; BMI, body mass index; FLI, fatty liver index; GGT, gamma-glutamyl transpeptidase; HOMA, homeostatic model assessment; IR, insulin resistance; LDL, low-density lipoprotein; LIG, low intensity exercise group; MET, metabolic equivalent; MIG, moderate intensity exercise group; NAFLD, non-alcoholic fatty liver disease.

Received: 10 March 2019; Revised: 27 December 2019; Accepted: 1 April 2020 *Correspondence to: Shivaram Prasad Singh, Department of Gastroenterology, SCB Medical College, Cuttack 753007, Orissa, India. Tel: +91-671-2505466, +91-671-2323624, E-mails: spsingh.cuttack@gmail.com; fattyliver@gmail.com terol $(216.8 \pm 29.2$ to $196.7 \pm 26.6 ; p=0.037)$, low-density lipoprotein cholesterol $(134.6 \pm 21.4$ to $130.5 \pm 21.9$; $p=0.010)$, serum aspartate transaminase $(39.3 \pm 32.2$ to $30.9 \pm 11.4 ; p<0.001)$, serum alanine transaminase $(56.6$ \pm 28.7 to $33.0 \pm 11.3 ; p<0.001)$ and HOMA-IR $(2.63 \pm 2.66$ to $1.70 \pm 2.59 ; p<0.001)$ in the MIG. However, changes in these parameters in the LIG were non-significant. Hepatic steatosis regressed in $66.7 \%$ of the NAFLD subjects in the MIG but in only $26.3 \%$ of the LIG NAFLD subjects $(p=0.030)$. Conclusions: Moderate rather than low intensity physical activity causes significant improvement in BMI, serum triglycerides, cholesterol, serum transaminases and HOMA-IR, and regression of ultrasonographic fatty change in liver among NAFLD subjects.

Citation of this article: Nath $P$, Panigrahi MK, Sahu MK, Narayan J, Sahoo RK, Patra AA, et al. Effect of exercise on NAFLD and its risk factors: Comparison of moderate versus low intensity exercise. J Clin Transl Hepatol 2020;8(2):120-126. doi: 10.14218/JCTH.2019.00012.

\section{Introduction}

Non-alcoholic fatty liver disease (NAFLD) is one of the most common hepatic disorders, with macrovesicular fat accumulation in more than $5 \%$ of hepatocytes in the absence of any secondary cause of hepatic steatosis, such as significant alcohol abuse (more than $10 \mathrm{~g} /$ day for women and $20 \mathrm{~g}$ /day for men), hepatotropic viral infection, drugs which can cause fatty liver, or any other etiologies. ${ }^{1}$ In the last couple of decades, NAFLD has emerged as the most common liver disease in adults. ${ }^{2}$ Its prevalence varies in different parts of the world, ranging from 5-40\%. However, its occurrence is even higher in individuals with obesity (30-100\%) and type 2 diabetes mellitus (42.6-69.5\%). ${ }^{3}$ Despite the benign natural course in the majority, $10 \%$ of the NAFLD patients may progress to cirrhosis later on in their lives. ${ }^{4}$ It contributes to formation of a pro-inflammatory environment that accelerates atherosclerosis, increasing the risk of ischemic heart disease and its severity, which itself correlates with the degree of inflammation. ${ }^{5}$ In addition, these individuals are at a high risk for developing diabetes mellitus. Hence, patient education about the entity, need for treatment and compliance are necessary to avert future complications. 
Lifestyle modification is still the treatment of first choice for NAFLD patients and is recommended for all patients with NAFLD. ${ }^{6}$ This aims at weight loss and reduction of insulin resistance (IR). Further, it is also directed at managing obesity and the features of metabolic syndrome which are frequently associated with NAFLD. Low-calorie diet and increased physical activity are the cornerstones of lifestyle modification. Patients are encouraged to lose at least $10 \%$ of their initial body weight to achieve maximal benefits. Weight loss improves patients' cardiovascular risk profile and steatosis, $^{7}$ and probably reduces hepatic inflammation as well as hepatocellular injury. ${ }^{8}$ Exercise is a major component of treatment for NAFLD, as recommended by the American Gastroenterological Association, ${ }^{9}$ the American Association for the Study of Liver Diseases and the European Association of Study of Liver. As compared to type 2 diabetes mellitus, there is a paucity of data supporting the role of physical activity in management of NAFLD. This may be due to the invasive nature of grading hepatic steatosis by needle biopsy and histology, which limits the capacity for repeated measurement of hepatic steatosis and degree of necroinflammation. The federal guidelines of the USA's Department of Health and Human Services and the USA's Department of Agriculture recommend that adults should perform 150 min or more of moderate-intensity physical activity per week, 75 min or more of vigorous intensity physical activity per week, or a combination, to improve and maintain health. ${ }^{10}$ The Centers for Disease Control and Prevention and the World Health Organization ${ }^{11}$ recommendations are also the same. For additional health benefits, the amount of physical activity recommended should be doubled. ${ }^{11}$ However, controversy remains over the role of exercise intensity and total volume of exercise responsible for final health outcomes.

The aim of this study was to compare the beneficial effects of low and moderate intensity exercise for 6 months, on NAFLD.

\section{Methods}

This study was performed in the Biju Patnaik State Police Academy, Bhubaneswar, Odisha, India which conducts 6 months of physical training before recruitment or promotion. The subjects were middle-aged male police recruits selected for 6 months of physical training.

\section{Inclusion criteria}

The police trainees who had fatty liver detected on ultrasonography were included in this study. These participants were in good health and had no abnormal findings on general and systemic physical examinations and for blood count and transabdominal ultrasonography (except for fatty liver).

\section{Exclusion criteria}

Patients with organic gastrointestinal disease revealed by ultrasonography or gastroduodenoscopy were excluded. Participants who had history of alcohol consumption exceeding $20 \mathrm{~g} /$ day, subjects with other liver diseases [hepatitis viruses A through E (by viral serologies: hepatitis B surface antigen, anti-hepatitis $C$ virus antibody), autoimmune disease (autoimmune markers: anti-nuclear antibody, anti-smooth muscle antibody, anti-liver kidney microsome type 1 antibody) and Wilson's disease (serum ceruloplasmin)] and those on drugs which can induce fatty liver or insulin sensitization (estrogens, amiodarone, methotrexate, tamoxifen, glitazones, metformin) were also excluded from the study.

An informed consent was obtained from each participant. Detailed anthropometric assessment, including measurements of weight, height, and waist and hip circumferences, was conducted before and after 6 months of physical exercise. The waist circumference was measured at a level midway between the lowest rib and the iliac crest and hip circumference at the level of the greater trochanter. Body mass index (BMI) was calculated by the formula of weight $(\mathrm{kg}) /$ height $^{2}\left(\mathrm{~m}^{2}\right)$.

The measurements of fasting plasma glucose, $2-\mathrm{h}$ postglucose load plasma glucose, serum triglycerides, serum total cholesterol, serum high-density lipoprotein cholesterol and liver function markers were performed by standard laboratory methods both before the beginning and after the completion of 6 months of physical exercise. The estimation of serum insulin was performed by using the electrochemiluminescence method (Roche-Diagnostics, USA) with an autoanalyzer, Elecsys 2010 (Roche-Hitachi, Japan). IR was computed by using the homeostatic model assessment (HOMA) method via a mathematical model derived from fasting plasma glucose and plasma insulin. The value of HOMA was calculated by the following equation: [fasting insulin $(\mu \mathrm{U} / \mathrm{mL}) \times$ fasting blood glucose $(\mathrm{mg} / \mathrm{dL})] / 405$, and depicted as the HOMA-IR value. ${ }^{12}$ For the purpose of the study, a HOMA-IR value above 2 was considered to indicate IR. ${ }^{13}$

Transabdominal ultrasonography was performed independently and blindly by two experienced radiologists (AAP and SJ; 5-10 years' experience) to identify and grade fatty changes in liver; in case of any conflict, the discrepancy was resolved by consensus after examination by a third senior radiologist (RKS; 15 years' experience). The diagnosis of fatty liver was made as per the standard criteria adopted by the American Gastroenterology Association, ${ }^{14}$ i.e. an increase in liver echogenicity as compared to renal echogenicity as a reference, as well as lack of differentiation in periportal intensity and vascular wall due to hyperechogenicity of the liver parenchyma.

All the participants were subjected to aerobic physical training for 6 months, which included brisk walking, jogging, marching drill, 'lathi' drill, and yoga. The duration of each session of the physical activity was 50-60 m per day with a frequency of 5-6 sessions per week. The duration of physical activity and total calories burnt during exercise (volume of exercise) were estimated by accelerometers. The intensity of exercise was deducted by the equation for metabolic equivalent (MET) as follows: (total calories)/(kg body weight)/(hours of exercised performed). Activities with MET values below 3 were labelled as low intensity exercise, those with MET between 3 and 5.9 were classified as moderate intensity, and activities with MET values $\geq 6$ were classified as vigorous intensity.

The subjects of the study were classified into the following groups:

1. Low intensity exercise group (LIG) and

2. Moderate intensity exercise group (MIG).

None of the study participants were subjected to vigorous intensity exercise. All the trainees were provided with a diet of 2400 to $2700 \mathrm{kcal} /$ day. This study was approved by the Institutional Ethics Committee of SCB Medical College, Cuttack.

\section{Statistical analysis}

Normally distributed continuous variables were expressed as mean \pm standard deviation. Student's $t$-test for unpaired data 


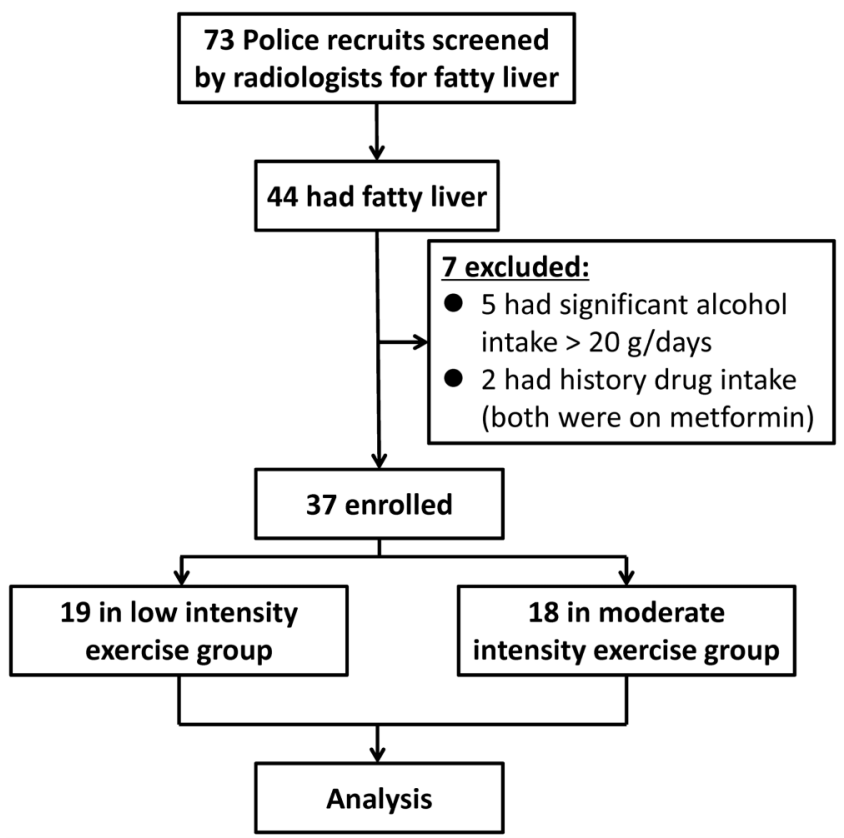

Fig. 1. Study consort diagram.

was used to compare the groups when the variables were normally distributed. Chi-square test was used to compare differences between categorical variables. All the anthropometric and biochemical parameters before and after 6 months of physical activity were compared by Student's $t$-test for paired data. All calculations were performed using the statistical software, SPSS version 16. A $p$ value of less than 0.05 was taken as significant.

\section{Results}

Out of 62 police recruits, a total of 37 subjects were to found to have NAFLD, out of which 18 performed moderate intensity exercise and 19 performed low intensity exercise for 6 months (Fig. 1). Baseline comparison between the two groups is depicted in Table 1 . The mean intensity of physical activity in the MIG was 3.6 MET (with mean total volume of exercise: 261.8 Kcalorie), whereas that of the LIG was 2.1 MET (with mean volume: 156.6 Kcalorie).

Patients in the LIG were older $(53.3 \pm 3.7$ years vs. 37.3 \pm 8.2 years; $p<0.001)$, with significantly higher systolic blood pressure $(156.6 \pm 18.9 \mathrm{mmHg}$ vs. $132.2 \pm 13.2 \mathrm{mmHg}$;

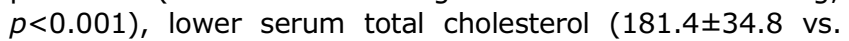
$211.2 \pm 30.0 ; p=0.018$ ), lower low-density lipoprotein (LDL) cholesterol $(102.5 \pm 29.9$ vs. $30.5 \pm 22.0 ; p=0.007)$, lower serum aspartate transaminase (AST; $23.9 \pm 7.9$ vs. 39.2 $\pm 29.5 ; p=0.039)$ and lower serum alanine transaminase (ALT; $26.3 \pm 13.1$ vs. $61.8 \pm 30.2 ; p<0.001$ ). However, both of the groups had comparable BMI, diastolic blood pressure, fasting and 2-h post-glucose load plasma glucose, serum triglycerides, high-density lipoprotein, very low-density lipoprotein, bilirubin, alkaline phosphatase, gamma-glutamyl

Table 1. Baseline characteristics of NAFLD subjects before 6 months of physical training

\begin{tabular}{|c|c|c|c|}
\hline Parameters & Low intensity exercise, $n=19$ & Moderate intensity exercise, $n=18$ & $p$ value \\
\hline Age (years) & $53.3 \pm 3.7$ & $37.3 \pm 8.2$ & $<0.001$ \\
\hline BMI $\left(\mathrm{kg} / \mathrm{m}^{2}\right)$ & $26.1 \pm 2.1$ & $26.9 \pm 1.9$ & 0.257 \\
\hline SBP (mm Hg) & $156.6 \pm 18.9$ & $132.2 \pm 13.2$ & $<0.001$ \\
\hline DBP (mm Hg) & $90.7 \pm 10.1$ & $87.4 \pm 8.3$ & 0.339 \\
\hline FPG (mg/dL) & $183.7 \pm 79.2$ & $104 \pm 20.7$ & 0.224 \\
\hline PGPG (mg/dL) & $185.8 \pm 67.4$ & $145.2 \pm 60.2$ & 0.090 \\
\hline Triglycerides (mg/dL) & $186.2 \pm 72.6$ & $157.9 \pm 57.2$ & 0.248 \\
\hline Cholesterol (mg/dL) & $181.4 \pm 34.8$ & $211.2 \pm 30.0$ & 0.018 \\
\hline HDL $(\mathrm{mg} / \mathrm{dL})$ & $42.1 \pm 8.9$ & $46.0 \pm 4.8$ & 0.159 \\
\hline LDL (mg/dL) & $102.5 \pm 29.9$ & $130.5 \pm 22.0$ & 0.007 \\
\hline VLDL (mg/dL) & $36.8 \pm 14.5$ & $34.6 \pm 16.8$ & 0.669 \\
\hline Bilirubin (mg/dL) & $1.1 \pm 0.7$ & $0.8 \pm 0.3$ & 0.225 \\
\hline AST (U/L) & $23.9 \pm 7.9$ & $39.2 \pm 29.5$ & 0.039 \\
\hline ALT (U/L) & $26.3 \pm 13.1$ & $61.8 \pm 30.2$ & $<0.001$ \\
\hline ALP (U/L) & $189.6 \pm 37.4$ & $202.7 \pm 51.3$ & 0.409 \\
\hline GGT (U/L) & $52.7 \pm 49.6$ & $61.3 \pm 51.3$ & 0.639 \\
\hline HOMA IR & $3.75 \pm 5.27$ & $2.56 \pm 2.55$ & 0.458 \\
\hline Exercise duration (minutes) & $57.21 \pm 4.22$ & $56.57 \pm 6.87$ & 0.728 \\
\hline Total calories burnt in exercise & $153.58 \pm 29.10$ & $261.81 \pm 37.18$ & $<0.001$ \\
\hline MET & $2.11 \pm 0.27$ & $3.61 \pm 0.42$ & $<0.001$ \\
\hline
\end{tabular}

Abbreviations: BMI, body mass index; SBP, systolic blood pressure; DBP, diastolic blood pressure; FPG, fasting plasma glucose; PGPG, post-glucose load plasma glucose (after 2-h glucose load); HDL, high density lipoprotein; LDL, low density lipoprotein; VLDL, very low density lipoprotein; AST, aspartate transaminase; ALT, alanine transaminase; ALP, alkaline phosphatase; GGT, gamma-glutamyl transpeptidase; HOMA-IR, homeostatic model assessment of insulin resistance; MET, metabolic equivalent. 
transpeptidase (GGT), HOMA-IR, 24-h dietary intake, and total duration of physical activity. On the other hand, subjects with moderate intensity exercise had significantly higher total calories burnt in exercise (261.81 $\$ 37.18$ Kcalorie vs. 153.58 \pm 29.10 Kcalorie; $p<0.001$ ).

In the LIG, there was significant decrease in waist hip ratio and waist height ratio, whereas decrease in mean BMI was modest $(0.2)$ and non-significant $(p=0.623)$ (Table 2). Besides, there was a significant reduction in blood pressure, serum total, high-density lipoprotein, very low-density lipoprotein cholesterol, and serum GGT. However, changes in all other parameters, including serum transaminases (AST and ALT), were non-significant. Furthermore, only 5 out of 19 $(26.3 \%)$ had resolution of fatty change in liver on ultrasonography.

Similarly, for the subjects who were subjected to moderate intensity exercise-although they showed modest improvement in BMI, waist hip ratio and waist height ratio, these changes were non-significant. However, there was significant reduction in blood pressure (Table 2 ). Further, there was significant improvement in lipid profile (except LDL cholesterol) and serum GGT. Unlike the LIG, subjects with moderate intensity exercise had elevated serum ALT at the baseline, and there were significant drops in serum levels of the transaminases (both AST and ALT) and decrease in HOMA-IR. Similarly, fasting glucose and triglycerides were normal/ borderline normal at baseline in the MIG, which decreased significantly after intervention; whereas, in the LIG, these parameters were abnormal, with no significant change. Moreover, the fatty changes in liver disappeared in 12 out 18 $(66.7 \%)$ subjects. The percentage of non-significant $(p>0.05)$ decrease in weight was higher in those who achieved resolution of fatty change in liver (Table 3 ).

Univariate logistic regression analysis was performed to assess the risk factors for resolution of fatty change in liver after physical training (Table 4). Out of all demographic, anthropometric, biochemical parameters and exercise indices, serum triglycerides, very low-density lipoprotein, presence of metabolic syndrome and exercise intensity were found to be significant predictors for response (sonographic resolution of fatty change in liver) to physical exercise in NAFLD participants. A multivariate logistic regression analysis was performed including the above risk factors (Table 4) and only exercise intensity was found to be an independent factor for resolution of fatty changes in liver on ultrasound. The noninvasive scores of fibrosis [NAFLD fibrosis score, fibrosis-4 score, and AST to platelet ratio (commonly referred to as APRI)] as well as fatty liver index (FLI) were computed for all study subjects. Participants in the MIG showed significant improvement in APRI score $(p=0.005)$ and FLI $(p<0.001)$ (Table 5). However, in the subjects belonging to the LIG, no significant improvement was observed in any of the scores, except for reduction in FLI $(p=0.031)$ after physical training (Table 5).

Table 2. Changes of parameters after 6 months of physical training in the low intensity exercise group and moderate intensity exercise group

\begin{tabular}{|c|c|c|c|c|c|c|}
\hline \multirow[b]{2}{*}{ Parameters } & \multicolumn{3}{|c|}{ Low intensity exercise group $(n=19)$} & \multicolumn{3}{|c|}{ Moderate intensity exercise group $(n=18)$} \\
\hline & $\begin{array}{l}\text { Before } 6 \text { months } \\
\text { of physical } \\
\text { training }\end{array}$ & $\begin{array}{l}\text { After } 6 \text { months } \\
\text { of physical } \\
\text { training }\end{array}$ & $p$ value & $\begin{array}{l}\text { Before } 6 \text { months } \\
\text { of physical } \\
\text { training }\end{array}$ & $\begin{array}{l}\text { After } 6 \text { months } \\
\text { of physical } \\
\text { training }\end{array}$ & $p$ value \\
\hline BMI $\left(\mathrm{kg} / \mathrm{m}^{2}\right)$ & $26.1 \pm 2.1$ & $25.9 \pm 1.8$ & 0.623 & $26.9 \pm 1.9$ & $26.7 \pm 2.0$ & 0.428 \\
\hline Waist hip ratio & $1.01 \pm 0.35$ & $0.93 \pm 0.22$ & $<0.001$ & $0.97 \pm 0.04$ & $0.96 \pm 0.04$ & 0.262 \\
\hline Waist height ratio & $0.59 \pm 0.04$ & $0.57 \pm 0.4$ & $<0.001$ & $0.56 \pm 0.04$ & $0.55 \pm 0.04$ & 0.168 \\
\hline SBP (mm Hg) & $156.6 \pm 18.9$ & $140.1 \pm 21.3$ & $<0.001$ & $132.2 \pm 13.2$ & $116.4 \pm 8.9$ & 0.001 \\
\hline DBP (mm Hg) & $90.7 \pm 10.1$ & $85.8 \pm 11.1$ & 0.018 & $87.4 \pm 8.3$ & $77.8 \pm 8.6$ & 0.005 \\
\hline FPG (mg/dL) & $183.7 \pm 79.2$ & $129.7 \pm 48.3$ & 0.333 & $104 \pm 20.7$ & $85.8 \pm 19.0$ & $<0.001$ \\
\hline PGPG (mg/dL) & $185.8 \pm 67.4$ & $185.1 \pm 85.5$ & 0.961 & $145.2 \pm 60.2$ & $120.1 \pm 40.8$ & 0.068 \\
\hline Triglycerides (mg/dL) & $186.2 \pm 72.6$ & $166.8 \pm 77.8$ & 0.303 & $157.9 \pm 57.2$ & $124.6 \pm 63.5$ & 0.013 \\
\hline Cholesterol (mg/dL) & $181.4 \pm 34.8$ & $167.1 \pm 33.2$ & 0.009 & $211.2 \pm 30.0$ & $196.7 \pm 26.6$ & 0.020 \\
\hline HDL (mg/dL) & $42.1 \pm 8.9$ & $37.8 \pm 6.7$ & 0.001 & $46.0 \pm 4.8$ & $41.5 \pm 6.8$ & 0.006 \\
\hline LDL (mg/dL) & $102.5 \pm 29.9$ & $99.4 \pm 26.6$ & 0.571 & $130.5 \pm 22.0$ & $130.5 \pm 21.9$ & 0.398 \\
\hline VLDL (mg/dL) & $36.8 \pm 14.5$ & $29.8 \pm 11.6$ & 0.032 & $34.6 \pm 16.8$ & $24.8 \pm 12.7$ & 0.010 \\
\hline Bilirubin (mg/dL) & $1.1 \pm 0.7$ & $0.9 \pm 0.8$ & 0.133 & $0.8 \pm 0.3$ & $0.7 \pm 0.4$ & 0.190 \\
\hline AST (U/L) & $23.9 \pm 7.9$ & $22.0 \pm 5.7$ & 0.204 & $39.2 \pm 29.5$ & $29.8 \pm 8.5$ & 0.253 \\
\hline ALT (U/L) & $26.3 \pm 13.1$ & $22.6 \pm 6.6$ & 0.205 & $61.8 \pm 30.2$ & $29.1 \pm 9.8$ & 0.002 \\
\hline ALP (U/L) & $189.6 \pm 37.4$ & $176.3 \pm 18.3$ & 0.137 & $202.7 \pm 51.3$ & $193.2 \pm 50.9$ & 0.440 \\
\hline GGT (U/L) & $52.7 \pm 49.6$ & $36.7 \pm 25.7$ & 0.033 & $61.3 \pm 51.3$ & $37.4 \pm 24.1$ & 0.024 \\
\hline HOMA-IR & $3.75 \pm 5.27$ & $2.71 \pm 2.60$ & 0.466 & $2.56 \pm 2.55$ & $1.69 \pm 2.59$ & 0.005 \\
\hline
\end{tabular}

Abbreviations: BMI, body mass index; SBP, systolic blood pressure; DBP, diastolic blood pressure; FPG, fasting plasma glucose; PGPG, post-glucose load plasma glucose (after 2-h glucose load); HDL, high density lipoprotein; LDL, low density lipoprotein; VLDL, very low density lipoprotein; AST, aspartate transaminase; ALT, alanine transaminase; ALP, alkaline phosphatase; GGT, gamma-glutamyl transpeptidase; HOMA-IR, homeostatic model assessment of insulin resistance. 
Nath P. et al: Effect of exercise intensity on NAFLD

Table 3. Comparison of percentage of change in weight in the study groups

\begin{tabular}{llll}
\hline & $\begin{array}{l}\text { Subjects with regression } \\
\text { of fatty liver }\end{array}$ & \multicolumn{2}{l}{$\begin{array}{l}\text { Subjects without regression } \\
\text { of fatty liver }\end{array}$} \\
\hline All subjects & $-2.28 \%$ & $-1.03 \%$ & 0.305 \\
Low intensity exercise group & $-0.20 \%$ & $-0.11 \%$ & 0.946 \\
Moderate intensity exercise group & $-3.19 \%$ & $-2.97 \%$ & 0.916 \\
\hline
\end{tabular}

Table 4. Logistic (univariate and multivariate) regression analysis of factors predicting resolution of fatty changes in liver in NAFLD subjects ( $n=37$ )

\begin{tabular}{|c|c|c|c|c|c|c|}
\hline Factors & OR & $95 \% \mathrm{CI}$ & $p$ value & AOR & $95 \% \mathrm{CI}$ & $p$ value \\
\hline Age (years) & 1.02 & $0.93-1.12$ & 0.673 & 1.07 & $0.92-1.26$ & 0.383 \\
\hline BMI $\left(\mathbf{k g} / \mathbf{m}^{2}\right)$ & 1.02 & $0.73-1.43$ & 0.908 & & & \\
\hline Waist circumference & 0.94 & $0.84-1.06$ & 0.302 & & & \\
\hline Waist hip ratio & 0.002 & $0.0001-1546$ & 0.443 & & & \\
\hline Waist height ratio & 0.54 & $0.0001-73566$ & 0.942 & & & \\
\hline SBP (mm Hg) & 0.98 & $0.95-1.01$ & 0.262 & & & \\
\hline DBP (mm Hg) & 1.01 & $0.93-1.07$ & 0.985 & & & \\
\hline FPG (mg/dL) & 0.98 & $0.96-1.01$ & 0.057 & & & \\
\hline PGPG (mg/dL) & 0.99 & $0.98-1.00$ & 0.106 & & & \\
\hline Triglycerides (mg/dL) & 0.98 & $0.96-0.99$ & 0.009 & 0.58 & $0.26-1.31$ & 0.189 \\
\hline Cholesterol (mg/dL) & 1.01 & $0.98-1.03$ & 0.631 & & & \\
\hline HDL (mg/dL) & 1.04 & $0.95-1.14$ & 0.362 & & & \\
\hline LDL $(\mathrm{mg} / \mathrm{dL})$ & 1.02 & $0.99-1.04$ & 0.158 & & & \\
\hline VLDL (mg/dL) & 0.90 & $0.83-0.98$ & 0.012 & 13.84 & $0.24-80.79$ & 0.205 \\
\hline Bilirubin (mg/dL) & 0.26 & $0.02-3.51$ & 0.311 & & & \\
\hline AST (U/L) & 1.03 & $0.98-1.08$ & 0.324 & & & \\
\hline ALT (U/L) & 1.01 & $0.98-1.04$ & 0.340 & & & \\
\hline $\operatorname{ALP}(U / L)$ & 1.01 & $0.99-1.02$ & 0.457 & & & \\
\hline GGT (U/L) & 1.01 & $0.99-1.02$ & 0.490 & & & \\
\hline Fasting insulin & 0.87 & $0.69-1.07$ & 0.191 & & & \\
\hline HOMA-IR & 0.52 & $0.24-1.11$ & 0.089 & & & \\
\hline Metabolic Syndrome & 4.29 & $1.06-17.36$ & 0.041 & 0.910 & $0.13-6.55$ & 0.926 \\
\hline Total duration of exercise & 1.01 & $0.89-1.29$ & 0.978 & & & \\
\hline Total calories burnt during exercise & 1.01 & $0.98-1.02$ & 0.122 & & & \\
\hline Exercise intensity (low vs. moderate) & 0.18 & $0.04-0.74$ & 0.017 & 17.18 & $1.16-253.61$ & 0.038 \\
\hline MET & 2.35 & $1.01-5.47$ & 0.043 & & & \\
\hline
\end{tabular}

Abbreviations: BMI, body mass index; SBP, systolic blood pressure; DBP, diastolic blood pressure; FPG, fasting plasma glucose; PGPG, post-glucose load plasma glucose (after 2-h glucose load); HDL, high density lipoprotein; LDL, low density lipoprotein; VLDL, very low density lipoprotein; AST, aspartate transaminase; ALT, alanine transaminase; ALP, alkaline phosphatase; GGT, gamma-glutamyl transpeptidase; HOMA-IR, homeostatic model assessment of insulin resistance; MET, metabolic equivalent; OR, odds ratio; AOR, adjusted odds ratio; $95 \% \mathrm{CI}$ : $95 \%$ confidence interval.

\section{Discussion}

The MIG had an elevated ALT and borderline normal fasting glucose and serum triglycerides at baseline, with significant improvement in these parameters at the end of the 6-month period. However, the LIG had normal ALT and abnormal fasting glucose and serum triglycerides at baseline but no significant change after intervention. The basic principle underlying use of physical exercise in treatment of NAFLD is improvement in insulin sensitivity. Moderate to vigorous physical activity leads to reduction in total body fat, especially in visceral adipose tissue. ${ }^{15}$ Decreased visceral adiposity leads to reduced fatty acid delivery to the liver, thereby decreasing hepatic steatosis and resultant necroinflammation.

In our study, moderate intensity aerobic exercise resulted in significant resolution of fatty change in liver and improvement in serum aminotransferases (AST and ALT). The role of physical activity in NAFLD has been investigated in various studies. Suzuki et al. ${ }^{16}$ demonstrated that regular exercise was associated with ALT reduction. Further, for every $5 \%$ in 
Nath P. et al: Effect of exercise intensity on NAFLD

Table 5. Non-invasive fibrosis scores of NAFLD and FLI before and after exercise

\begin{tabular}{|c|c|c|c|c|c|c|}
\hline \multirow{2}{*}{ Score } & \multicolumn{3}{|c|}{ Moderate intensity group } & \multicolumn{3}{|c|}{ Low intensity group } \\
\hline & Before exercise & After exercise & $p$ value & Before exercise & After exercise & $p$ value \\
\hline NFS & -1.35 & -1.59 & 0.278 & 0.66 & 0.75 & 0.621 \\
\hline FIB-4 & 0.84 & 0.81 & 0.722 & 0.88 & 0.86 & 0.772 \\
\hline APRI & 0.41 & 0.27 & 0.005 & 0.43 & 0.40 & 0.285 \\
\hline FLI & 62.84 & 50.55 & $<0.001$ & 66.08 & 60.43 & 0.031 \\
\hline
\end{tabular}

Abbreviations: NAFLD, non-alcoholic fatty liver disease; NFS, NAFLD fibrosis score; FIB-4, fibrosis-4 score; APRI, aspartate transaminase to platelet ratio index; FLI, fatty liver index.

weight loss, a 3.6 greater likelihood of ALT normalization was observed in their study. ${ }^{16}$ Studies employing magnetic resonance spectroscopy (known as $1 \mathrm{H}$-MRS) also confirm these reports. ${ }^{17,18}$ In our study, although the degree of weight loss in both the groups was not significant, there was a significant fall in serum transaminases in the MIG. However, in the LIG, the reduction in transaminases was not significant.

The effect of exercise with or without hypocaloric diet on NAFLD patients has been studied in various clinical trials. ${ }^{8,19-27}$ Most of these studies had smaller number of subjects, absence of measurement of volume (calorie) and intensity of exercise, and some lacked histological endpoints. A comparison of the results of eight studies, including the present study, is shown in Supplementary Table 1. All trials showed significant improvement in BMI, serum enzymes (AST/ALT), and degree of fatty liver. There are several studies that have demonstrated a dose-dependent improvement in liver histology ${ }^{27}$ and intrahepatic triglycerides ${ }^{26}$ dependent on the degree of weight loss achieved. However, the improvement in transaminases, HOMA-IR, FLI and resolution of ultrasonographic fatty change in liver was independent of the degree of weight loss in our study. Further studies are required, with longer duration and with more intense physical activities, to demonstrate changes in fibrosis score.

Recently, the intensity rather than duration of exercise and total calorie expenditure during physical activity has caught the attention of researchers. Kistler et al. ${ }^{23}$ in a retrospective study examined the effect of exercise intensity on histological severity of NAFLD. In this study, the exercise volume and intensity was calculated by self-reported physical activity data from adult patients with biopsy-proven NAFLD enrolled in the Nonalcoholic Steatohepatitis Clinical Research Network, and the NAFLD patients were classified into moderate and vigorous exercise groups as per the federal recommendations. The study demonstrated an inverse relationship between the intensity of physical activity and severity of NAFLD. However, this study was limited by its cross-sectional nature, measurement limitations, and misclassification due to reporting and recall bias. On the contrary, in our study, we measured the exercise intensity prospectively with objective methods (by accelerometers).

Our study had several limitations. The two study groups were not entirely comparable as there were significant differences in age, baseline levels of serum AST and ALT, serum total cholesterol, and serum LDL cholesterol. Besides, the sample size was small and only male NAFLD subjects were included. Furthermore, the diagnosis of NAFLD was based on transabdominal ultrasound, which can miss fatty liver when the degree of steatosis is less than $30 \% .^{28}$

\section{Conclusions}

Moderate intensity of physical activities for a duration of 6 months helps in improvement in hepatic steatosis, serum transaminitis, glycemic and lipid profiles as well as IR, as compared with low intensity exercise. All individuals with NAFLD should perform moderately intense physical activities for maximum benefits. More rigorous, controlled studies, of longer duration and with defined histopathological end-points are the need of the hour for better evidence-based lifestyle modification guidelines.

\section{Funding}

This study was sponsored by the Kalinga Gastroenterology Foundation, Cuttack, Odisha (India).

\section{Conflict of interest}

The authors have no conflict of interests related to this publication.

\section{Author contributions}

Study concept and design (PN, SPS), acquisition of data (PN, MKS, JN, RKS, AAP, SJ, AKP, AJ), analysis and interpretation of data (PN, MKP, SPS), drafting of the manuscript (PN, MKP), critical revision of the manuscript for important intellectual content (PN, MKP, JN, SPS), study supervision (SPS).

\section{References}

[1] Angulo $P$, Lindor KD. Non-alcoholic fatty liver disease. J Gastroenterol Hepatol 2002;17:S186-S190. doi: 10.1046/j.1440-1746.17.s1.10.x.

[2] Vernon G, Baranova A, Younossi ZM. Systematic review: the epidemiology and natural history of non-alcoholic fatty liver disease and non-alcoholic steatohepatitis in adults. Aliment Pharmacol Ther 2011;34:274-285. doi: 10.1111/j.1365-2036.2011.04724.x.

[3] Blachier M, Leleu H, Peck-Radosavljevic M, Valla DC, Roudot-Thoraval F. The burden of liver disease in Europe: a review of available epidemiological data. J Hepatol 2013;58:593-608. doi: 10.1016/j.jhep.2012.12.005.

[4] Calzadilla Bertot L, Adams LA. The Natural course of non-alcoholic fatty liver disease. Int J Mol Sci 2016;17:774. doi: 10.3390/ijms17050774.

[5] Brea A, Puzo J. Non-alcoholic fatty liver disease and cardiovascular risk. Int ] Cardiol 2013;167:1109-1117. doi: 10.1016/j.ijcard.2012.09.085.

[6] Chalasani N, Younossi Z, Lavine JE, Diehl AM, Brunt EM, Cusi K, et al. The diagnosis and management of non-alcoholic fatty liver disease: practice Guideline by the American Association for the Study of Liver Diseases, American College of Gastroenterology, and the American Gastroenterological Association. Hepatology 2012;55:2005-2023. doi: 10.1002/hep.25762.

[7] Sullivan S, Kirk EP, Mittendorfer B, Patterson BW, Klein S. Randomized trial of exercise effect on intrahepatic triglyceride content and lipid kinetics in 
nonalcoholic fatty liver disease. Hepatology 2012;55:1738-1745. doi: 10 . 1002/hep.25548.

[8] Promrat K, Kleiner DE, Niemeier HM, Jackvony E, Kearns M, Wands JR, et al. Randomized controlled trial testing the effects of weight loss on nonalcoholic steatohepatitis. Hepatology 2010;51:121-129. doi: 10.1002/hep.23276.

[9] American Gastroenterological Association medical position statement: nonalcoholic fatty liver disease. Gastroenterology 2002;123:1702-1704. doi: $10.1053 /$ gast.2002.36569.

[10] US Department of Health and Human Services. Physical activity guidelines for Americans. 2nd edition. Available from: https://health.gov/sites/default/files/2019-09/Physical_Activity_Guidelines_2nd_edition.pdf.

[11] World Health Organization. Global recommendations on physical activity for health. Available from: http://apps.who.int/iris/bitstream/10665/44399/ 1/9789241599979_eng.pdf.

[12] Matthews DR, Hosker JP, Rudenski AS, Naylor BA, Treacher DF, Turner RC. Homeostasis model assessment: insulin resistance and beta-cell function from fasting plasma glucose and insulin concentrations in man. Diabetologia 1985;28:412-419. doi: 10.1007/bf00280883.

[13] Gayoso-Diz P, Otero-González A, Rodriguez-Alvarez MX, Gude F, García F, De Francisco $A$, et al. Insulin resistance (HOMA-IR) cut-off values and the metabolic syndrome in a general adult population: effect of gender and age: EPIRCE cross-sectional study. BMC Endocr Disord 2013;13:47. doi: 10. 1186/1472-6823-13-47.

[14] Gore RM. Diffuse liver disease. In: Gore R, Levine M, editors. Textbook of gastrointestinal radiology $3^{\text {rd }}$ Edition. Philadelphia: Saunders; 1994. P.19682017.

[15] Carroll JF, Franks SF, Smith AB, Phelps DR. Visceral adipose tissue loss and insulin resistance 6 months after laparoscopic gastric banding surgery: a preliminary study. Obes Surg 2009;19:47-55. doi: 10.1007/s11695-0089642-4.

[16] Suzuki A, Lindor K, St Saver J, Lymp J, Mendes F, Muto A, et al. Effect of changes on body weight and lifestyle in nonalcoholic fatty liver disease. J Hepatol 2005;43:1060-1066. doi: 10.1016/j.jhep.2005.06.008.

[17] Johnson NA, Sachinwalla T, Walton DW, Smith K, Armstrong A, Thompson $M W$, et al. Aerobic exercise training reduces hepatic and visceral lipids in obese individuals without weight loss. Hepatology 2009;50:1105-1112. doi: 10.1002/hep.23129.

[18] Hallsworth K, Fattakhova G, Hollingsworth KG, Thoma C, Moore S, Taylor R, et al. Resistance exercise reduces liver fat and its mediators in non-alcoholic fatty liver disease independent of weight loss. Gut 2011;60:1278-1283. doi: 10.1136/gut.2011.242073.
[19] Ueno T, Sugawara H, Sujaku K, Hashimoto O, Tsuji R, Tamaki S, et al. Therapeutic effects of restricted diet and exercise in obese patients with fatty liver. J Hepatol 1997;27:103-107. doi: 10.1016/s0168-8278(97)80287-5.

[20] Huang MA, Greenson JK, Chao C, Anderson L, Peterman D, Jacobson J, et al. One-year intense nutritional counseling results in histological improvement in patients with non-alcoholic steatohepatitis: a pilot study. Am J Gastroenterol 2005;100:1072-1081. doi: 10.1111/j.1572-0241.2005.41334.x.

[21] Nobili V, Manco M, Devito R, Di Ciommo V, Comparcola D, Sartorelli MR, et al. Lifestyle intervention and antioxidant therapy in children with nonalcoholic fatty liver disease: a randomized, controlled trial. Hepatology 2008;48:119128. doi: 10.1002/hep.22336.

[22] Vilar Gomez E, Rodriguez De Miranda A, Gra Oramas B, Arus Soler E, Llanio Navarro R, Calzadilla Bertot $\mathrm{L}$, et al. Clinical trial: a nutritional supplement Viusid, in combination with diet and exercise, in patients with nonalcoholic fatty liver disease. Aliment Pharmacol Ther 2009;30:999-1009. doi: 10 . 1111/j.1365-2036.2009.04122.x.

[23] Kistler KD, Brunt EM, Clark JM, Diehl AM, Sallis JF, Schwimmer JB. Physical activity recommendations, exercise intensity, and histological severity of nonalcoholic fatty liver disease. Am J Gastroenterol 2011;106:460-468. doi: 10.1038/ajg.2010.488.

[24] St George A, Bauman A, Johnston A, Farrell G, Chey T, George J. Independent effects of physical activity in patients with nonalcoholic fatty liver disease. Hepatology 2009;50:68-76. doi: 10.1002/hep.22940.

[25] Wong VW, Chan RS, Wong GL, Cheung BH, Chu WC, Yeung DK, et al. Community-based lifestyle modification programme for non-alcoholic fatty liver disease: a randomized controlled trial. J Hepatol 2013;59:536-542. doi: 10 1016/j.jhep.2013.04.013.

[26] Houghton D, Thoma C, Hallsworth K, Cassidy S, Hardy T, Burt AD, et al. Exercise reduces liver lipids and visceral adiposity in patients with nonalcoholic steatohepatitis in a randomized controlled trial. Clin Gastroenterol Hepatol 2017;15:96-102.e3. doi: 10.1016/j.cgh.2016.07.031.

[27] Vilar-Gomez E, Martinez-Perez Y, Calzadilla-Bertot L, Torres-Gonzalez A, Gra-Oramas B, Gonzalez-Fabian L, et al. Weight loss through lifestyle modification significantly reduces features of nonalcoholic steatohepatitis. Gastroenterology 2015;149:367-378.e5. doi: 10.1053/j.gastro.2015.04.005.

[28] Hernaez R, Lazo M, Bonekamp S, Kamel I, Brancati FL, Guallar E, et al. Diagnostic accuracy and reliability of ultrasonography for the detection of fatty liver: a meta-analysis. Hepatology 2011;54:1082-1090. doi: 10. 1002/hep.24452. 